\title{
A Dual Photochemical Ring-Opening/Cleavage Approach for the Synthesis and Decoding of One-Bead-One-Compound Cyclic Peptide Libraries
}

\author{
Xinxia Liang, Simon Vézina-Dawod, François Bédard, and Eric Biron \\ Faculty of Pharmacy, Université Laval and Laboratory of Medicinal Chemistry, CHU de Québec Research \\ Centre, CHUL, Quebec (QC), G1V 4G2, Canada
}

\section{Introduction}

With a great therapeutic potential, peptide macrocycles have gained a lot of interest in drug discovery. Compared to their linear counterparts, cyclic peptides show improved resistance to proteases and their increased conformational rigidity lowers the entropic cost of binding, making them tighter-binding to a given macromolecule $[1,2]$. The great degree of molecular diversity and complexity that can be accessed by simple changes in their sequence has prompted the use of cyclic peptides in combinatorial chemistry. The one-bead-one-compound (OBOC) approach, in which each bead carries many copies of a unique compound, has become a powerful tool in the drug discovery process [3]. However, the use of cyclic peptides in combinatorial OBOC libraries has been limited by difficulties in sequencing hit compounds after the screening. Lacking a free $\mathrm{N}$-terminal amine, Edman degradation sequencing cannot be used on cyclic peptides and complicated fragmentation patterns are obtained by tandem mass spectrometry (MS/MS). This problem has been initially overcome by using a bead topological segregation strategy [4,5]. More recently, different strategies have been reported to avoid encoding by using a ring-opening approach to allow a simultaneous linearization and compound release from the bead [6-9]. Most of the reported methods require post-screening chemical reactions that could lead to side chain modification. Based on these strategies, we were looking for an efficient, single step and chemical reagent free ring-opening approach that would be compatible with free amino acid side chains.

\section{Results and Discussion}

Our approach utilizes the photocleavable $\beta$-amino acid 3-amino-3-(2-nitrophenyl)propionic acid (ANP) as a linker and within the macrocycle (Figure 1). The strategy is to use UV irradiation to simultaneously convert cyclic peptides into their linear counterpart and release them from the beads. The generated linear peptides could then be sequenced by MS/MS. To evaluate the ring-opening upon UV irradiation, a model cyclic peptide with the sequence ANP-Leu-Gly-Tyr-Gly-Lys-Phe-Glu was first synthesized on Rink Amide AM polystyrene resin.

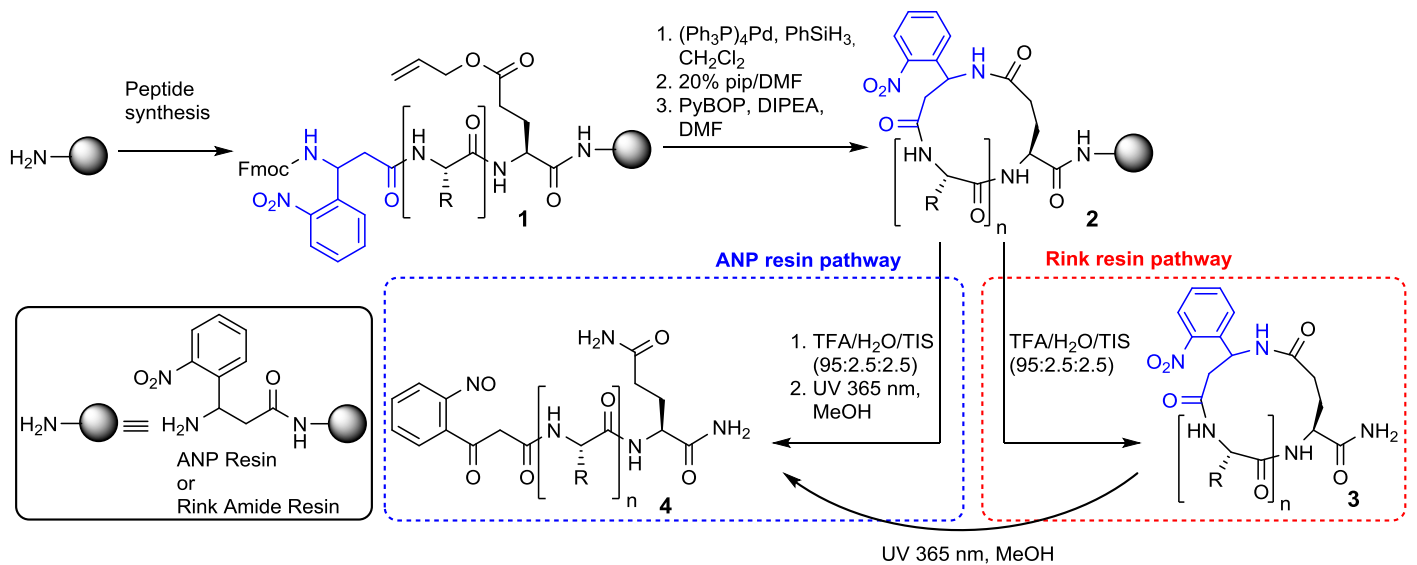

Fig. 1. Synthetic route to cyclic peptides with ANP residue and ring-opening upon UV irradiation. 
Linear peptide 1 was synthesized on solid support by standard Fmoc chemistry. After removing the allyl and Fmoc protecting groups, the peptide was cyclised on resin with PyBOP. After cyclization, the side chains protecting groups were removed and the compound cleaved from the resin with a TFA cocktail. The released cyclic peptide $\mathbf{3}$ was then analysed by HPLC and electrospray ionization MS (ESI-MS) to confirm the absence of linear peptide (Figure 2a). Cyclic peptide 3 was then subjected to UV-irradiation at $365 \mathrm{~nm}$ in MeOH for ring cleavage. HPLC analysis confirmed the transformation of the cyclic peptide into its linear counterpart 4 (Figure $2 \mathrm{~b}$ ). The observed main peak was a doublet with both peaks having the same mass. This result led us to presume the formation of two diastereomers during ring-opening. Since the cyclic and linearized peptides have exactly the same elemental composition, the use of MS to differentiate cyclic $\mathbf{3}$ from linear compound $\mathbf{4}$ is theoretically limited. Surprisingly, after MS analysis of the generated linear peptide 4, the anticipated molecular ion was not obtained and the most important peak was observed at $\mathrm{M}+\mathrm{H}^{+}+14 \mathrm{Da}$ (Figure $2 \mathrm{~b}$ ). This observation led us to suspect that the reaction did not stop at the nitroso product as expected. Further analysis of the photocleavage reaction mechanism of ortho-nitrophenyl derivatives, proposed that the $+14 \mathrm{Da}$ peak is generated during UV irradiation from the addition of $\mathrm{MeOH}(32 \mathrm{Da})$ on a dehydrated specie $\left(\mathrm{M}+\mathrm{H}^{+}-\right.$ 18) of the nitroso derivative 4. This hypothesis was supported by the following results: 1) when methanol-d4 (36 Da) was used as solvent, the adduct peak was observed at $+18 \mathrm{Da}$; and 2) only the M-18 peak was observed in absence of $\mathrm{MeOH}$ during MALDI-TOF MS analysis. Further investigations to understand this reaction are underway.

To evaluate the efficiency of the simultaneous ring-opening/cleavage strategy, the model cyclic peptide 2 was synthesized on TentaGel $\mathrm{S} \mathrm{NH}_{2}$ resin bearing the ANP as linker. After cyclization and side chains deprotection, a small amount of resin 2 was subjected to UV-irradiation and the released peptide analysed by HPLC and ESI-MS to confirm the presence of linear peptide 4 . Next, a single bead was picked up from the resin $\mathbf{2}$ and exposed to UV-irradiation in $\mathrm{MeOH}$. The crude product released from a single bead was immediately subjected to MALDI-TOF MS. Surprisingly, the mass spectra did not show the peak of the $\mathrm{MeOH}$ adduct $\left(\mathrm{M}+\mathrm{H}^{+}+14\right)$ but the dehydrated product $\left(\mathrm{M}-18+\mathrm{H}^{+}\right)$was observed as the major peak (Figure 3a). These results suggest that the adduct product is modified into the dehydrated specie when exposed to the laser during ionisation in the MALDI instrument. The ability of the MALDI's Nd:YAG laser to induce photochemical reaction has been shown and used by a few groups for synthesis monitoring and structural elucidation of biomolecules [10,11]. MS/MS analysis of the dehydrated product molecular ion yielded high-quality spectra from which the linearized peptide 4 could be unambiguously sequenced manually and by using de novo sequencing with the Peaks software (Figure 3b) [12].
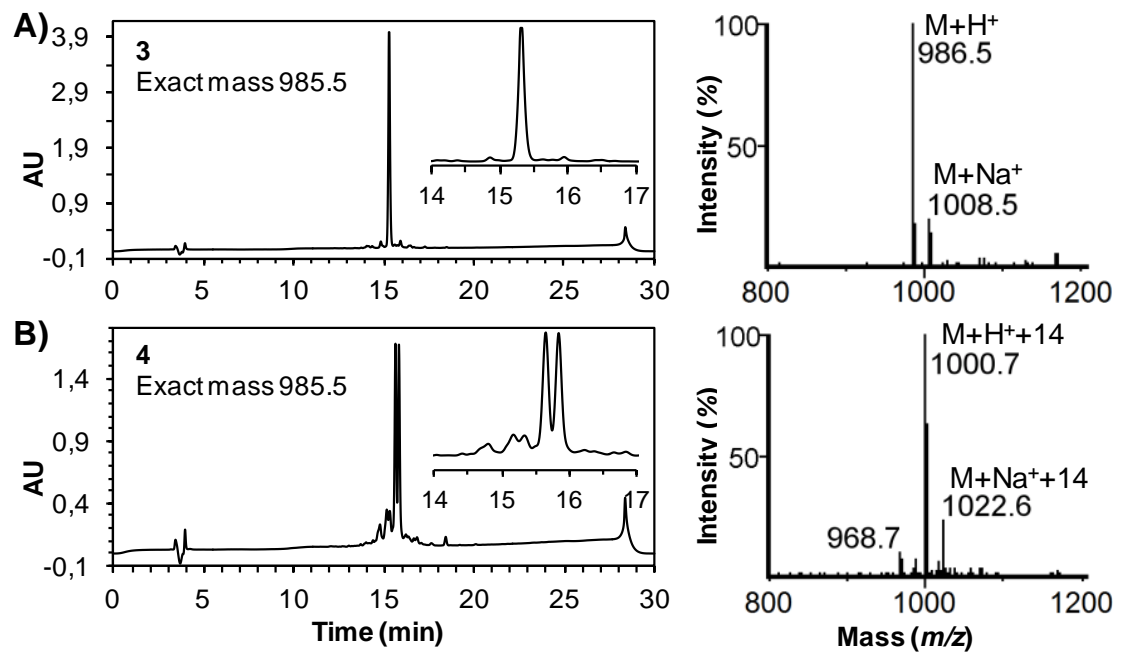

Fig. 2. HPLC and ESI-MS profiles of crude products showing cyclization and ring-opening. (A) cyclo[ANP-Leu-Gly-Tyr-Gly-Lys-Phe-Glu]-NH2 3. (B) 3-oxo-3-(2-nitrosophenyl)propionyl-Leu-GlyTyr-Gly-Lys-Phe-Gln-NH $\mathrm{H}_{2} 4$. 


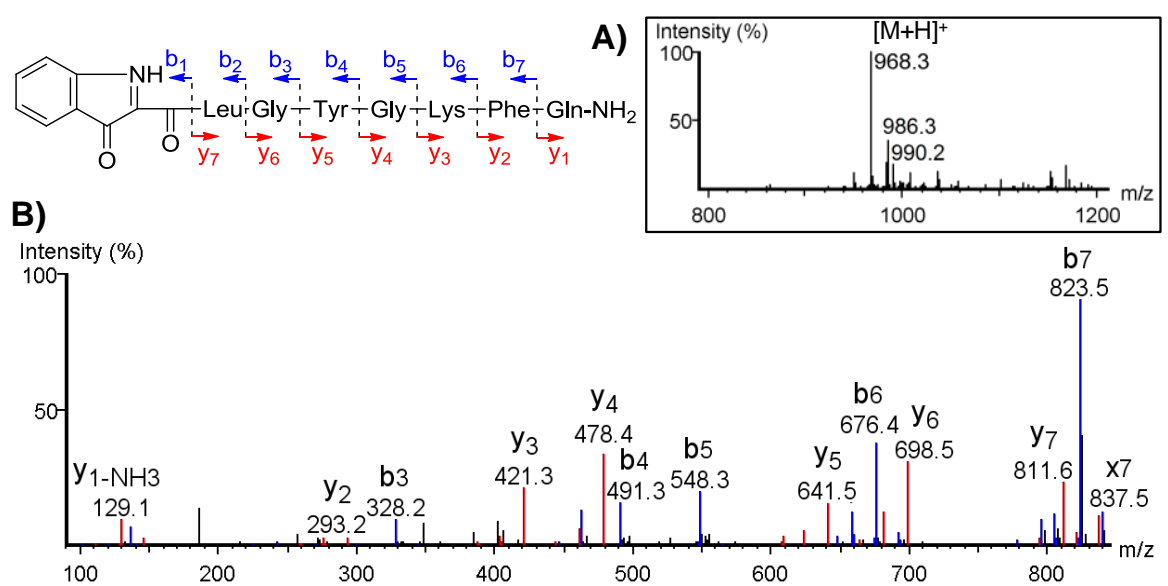

Fig. 3. MS and MS/MS spectra obtained after dual ring opening/cleavage on a single bead of cyclic peptide 2. (A) MS of the crude product; (B) MS/MS of the dehydrated specie $[M-18+H]^{+}(968.3 \mathrm{Da})$.

In summary, we report the use of a photolabile residue as a linker and within a cyclic peptide to allow a simultaneous ring-opening of the macrocycle and cleavage from the resin upon UV irradiation. The linearized peptides can then be efficiently sequenced by MS/MS analysis. This approach reduces the need for post-screening chemical modification for sequencing and allows a fast sequence determination of cyclic peptides from combinatorial libraries by MS/MS. As previously reported [6-9], compared to topological segregated bilayer beads, the re-opening strategy in OBOC libraries eliminates the risks of interference by the tag during screening since the same molecules are displayed inside and on the surface of the beads. Simple and affordable for any peptide science or combinatorial chemistry laboratory, the described procedure is compatible with commonly used amino acid side chains and could be readily used for the synthesis of cyclic peptoid or other peptidomimetic OBOC libraries. The compatibility of our strategy with OBOC library synthesis and screening is currently investigated.

\section{Acknowledgments}

Xinxia Liang, Simon Vézina-Dawod and François Bédard respectively thank the China Schloarship Council (CSC), the Fond d'enseignement et de recherche de la Faculté de pharmacie de l'Université Laval (FER) and the Fonds de recherche du Québec - Nature et technologies (FRQNT) for graduate scholarships. This work was supported by the National Sciences and Engineering Research Council of Canada (NSERC) (371503-2010).

\section{References}

1. Adessi, C., Soto, C. Curr. Med. Chem. 9, 963-978 (2002), http://dx.doi.org/10.2174/0929867024606731

2. Pauletti, G.M., et al. Adv. Drug Delivery Rev. 27, 235-256 (1997), http://dx.doi.org/10.1016/S0169409X(97)00045-8

3. Lam, K.S., Krchnak, V., Lebl, M. Chem. Rev. 97, 411-448 (1997), http://dx.doi.org/10.1021/cr9600114

4. Liu, R., Marik, J., Lam, K.S. J. Am. Chem. Soc. 124, 7678-7680 (2002), http://dx.doi.org/10.1021/ja026421t

5. Joo, S.H., et al. J. Am. Chem. Soc. 128, 13000-13009 (2006), http://dx.doi.org/10.1021/ja063722k

6. Lee, J.H., Meyer, A.M., Lim, H.S. Chem. Comm. 46, 8615-8617 (2010), http://dx.doi.org/10.1039/C0CC03272G

7. Simpson, L.S., Kodadek, T. Tetrahedron Lett. 53, 2341-2344 (2012), http://dx.doi.org/10.1016/j.tetlet.2012.02.112

8. Liang, X., Girard, A., Biron, E. ACS Comb. Sci. 15, 535-540 (2013), http://dx.doi.org/10.1021/co4000979

9. Lee, K.J., Lim, H.S. Org. Lett. 16, 5710-5713 (2014), http://dx.doi.org/10.1021/ol502788e

10. Fitzgerald, M.C., Harris, K., Shevlin, C.G., Siuzdak G., Bioorg. Med. Chem. Lett. 6, 979-982 (1996), http://dx.doi.org/10.1016/0960-894X(96)00152-7

11. St-Hilaire P.M., Lowary, T.L., Meldal, M., Bock, K. J. Am. Chem. Soc. 33, 13312-13320 (1998), http://dx.doi.org/10.1021/ja980387u

12. Ma, B., Lajoie, G. Rapid Commun. Mass Spectrom. 17, 2337-2342 (2003), http://dx.doi.org/10.1002/rcm.1196 